\title{
Philosophiques
}

\section{La société distincte}

\section{Christian Dufour}

Volume 19, numéro 2, automne 1992

Une nation peut-elle se donner la constitution de son choix?

URI : https://id.erudit.org/iderudit/027191ar

DOI : https://doi.org/10.7202/027191ar

Aller au sommaire du numéro

Éditeur(s)

Société de philosophie du Québec

ISSN

0316-2923 (imprimé)

1492-1391 (numérique)

Découvrir la revue

Citer ce document

Dufour, C. (1992). La société distincte. Philosophiques, 19(2), 55-61.

https://doi.org/10.7202/027191ar d'utilisation que vous pouvez consulter en ligne.

https://apropos.erudit.org/fr/usagers/politique-dutilisation/ 


\title{
LA SOCIÉTÉ DISTINCTE
}

\author{
par \\ Christian Dufour
}

\section{L'HÉRTTAGE DE MEECH}

Au lieu de construire à partir d'un souverainisme en paroles, au lieu de bâtir sur du sable, il faut travailler davantage à partir de ce que nous sommes réellement. Tout d'abord, s'habituer à utiliser les pouvoirs dont on dispose sous le regard de cet Anglais qui fait partie de nous, sans s'incliner devant lui, mais sans le nier non plus. Ensuite, s'approprier sans s'excuser les pouvoirs nécessaires pour bâtir la société distincte québécoise de demain, que le reste du Canada la reconnaisse ou non, que le reste du Canada le veuille ou non.

Je souhaiterais pour ma part que l'Assemblée nationale se déclare pleinement souveraine pour bâtir, non pas un Québec indépendant, mais bien la société distincte québécoise. A partir de là, on pourrait pousser la société distincte le plus loin qu'il sera possible de le faire de façon réaliste, sans exclure l'indépendance mais sans en faire une nécessité non plus. La souveraineté des péquistes, alliée à la société distincte des libéraux: cela peut être le début d'un nouveau consensus quant à un projet national québécois adapté à l'an 2000. Il śagirait du naturel complément au consensus dégagé en iggi par la Commission Bélanger-Campeau sur la démarche à adopter quant à la renégociation de la

I. Ce texte est extrait de Pour en finir avec la Conquête, prèsentement sous presses aux Editions Boréal. 
relation avec le reste du Canada. Après le contenant, le contenu; après la forme, la substance ${ }^{2}$.

Que les Québécois constituent à tout le moins une société distincte a fait l'objet d'un consensus exceptionnellement large à l'occasion du débat sur l'Accord du lac Meech. Un moment, cela allait de Gilles Vigneault à Jeanne Sauvé. Dans une société aussi divisée que la nôtre, un concept politique qui permet de ratisser aussi large comporte quelque chose d'irremplaçable. De façon réaliste, les Québécois peuvent compter réaliser plusieurs de leurs rêves, s'ils partent d'une réalité solide comme le béton, dont le caractère minimal fait paradoxalement la force. Alors que des appellations comme nation, ou même peuple, suscitent des réserves chez certains Québécois, il faut être de mauvaise foi pour nier que les Québécois constituent à tout le moins une société distincte.

Au départ, la société distincte, telle qu'elle apparaît dans Meech, possède un contenu minimal: une majorité française, une minorité anglaise, des liens avec le reste du Canada. Pour le reste, le concept se définit avant tout par sa frontière. Éminemment politique, il est institutionnel, il est global. Cest heureux. Car les recherches sur les identités nationales montrent bien qu'au-delà d'un contenu qui peut changer considérablement, ce qui importe avant tout pour le progrès d'une identité nationale, cest le maintien d'une frontière. Or, si le contenu de la société distincte reste pour l'instant minimal, il est clair que la frontière y est. C'est le Québec - et non les francophones, les autochtones, le Manitoba ou Terre-Neuve - qui constitue une société distincte.

Utilisée, développée, enrichie, cette sèche et froide notion sera davantage porteuse de pouvoir politique pour le Québec que les appellations plus èmotivement valorisantes de peuples ou de nations, non seulement dans le contexte canadien mais aussi sur le plan international. Loin d'être un problème, l'absence initiale de contenu constitua pour le Québec une chance, en faisant ressortir le côté fondamentalement politique de l'affaire. Positive, encore vierge, toute neuve, la société distincte a l'avenir devant elle, potentiellement riche de toute la dense réalité québécoise. Il est révélateur que les autochtones canadiens, auxquels personne ne nie l'appellation de nations, n'en auront de cesse que lorsqu'ils auront été reconnus comme constituant des sociétés distinctes. Cest qu'instinctivement, ils sentent bien que la

2. Christian Dufour, le défi québécois, Montréal, l'Hexagone, 1987. 
société distincte a l'avenir pour elle, forte du pouvoir politique que les Québécois y ont investi lors du débat sur l'Accord du lac Meech.

Sur leur teritoire, les Québécois ont intėrêt à se voir comme une société ne se définissant pas en fonction de son contenu, fût-ce la langue française. Dans le cadre d'une société distincte où les francophones constituent plus de $80 \%$ de la population, il est possible de reconnaître la composante historique anglaise du Québec, sans verser dans le bilinguisme institutionnel. Le Québec français n'a rien à gagner à affronter un fait anglais extérieur à lui, là où il est le plus puissant: au Canada, en Amérique du Nord.

La socièté distincte, les Québécois sont clairement tentés de se l'approprier, ne serait-ce que pour ironiser à son sujet. Pas de doute que, s'ils en veulent, elle leur appartient, que pour l'heure elle n'appartient qu'à eux, arrachée de haute lutte lors de la dure bataille de Meech. De façon analogue, l'idée toute nouvelle de la nation appartenait toute entière à la France révolutionnaire de l'an I, quand elle gagnait sa première bataille de Valmy, en I792. Sous prétexte que le Québec n'a pas été reconnu comme société distincte par l'ordre constitutionnel canadien, que celui-ci veut maintenant réduire cette notion à ses aspects culturels, sous prétexte que sur le plan sociologique les sociétés distinctes abondent, renoncer à un statut politique dans lequel on a tant investi et qui nous rallie, serait tout le contraire de la souveraineté. Ce serait faire acte de soumission envers un nouvel ordre constitutionnel canadien qui nie que notre différence collective a des conséquences politiques ${ }^{3}$. Ce serait renoncer à notre avenir.

\section{LE POTENTIEL INTERNATIONAI}

Le côté novateur de la société distincte sur le plan international, c'est qu'elle permet l'expression politique du phénomène national dans ce qu'il a d'irréductible et de positif, tout en le dépouillant des éléments ethniques et émotifs susceptibles d'empêcher des relations fécondes avec les nations environnantes: il est plus facile d'en venir aux mains pour une nation que pour une société distincte. Dans un contexte de plus

3. Christian Dufour, "If mal canadien ", sous la direction de Louis Balthazar, Guy Laforest et Vincent Lemieux, Le Québec et la restructuration du Canada, Enjeux et perspectives, Sillery, Editions du Septentrion, I99I, Pp. Iog120. 
grande intégration économique de l'ensemble des nations, dans un contexte de mondialisation, certains indices permettent d'escompter qu'en particulier dans le monde développé, la société distincte soit promise à un bel avenir. Dans un nombre croissant de cas, elle serait appelée à se substituer à la nation, de la même façon que celle-ci a remplacé l'État-monarchique, il y a deux cent ans.

Se gardant bien de se tromper de guerre ou d'époque, il faut regarder en avant, essayer de voir loin. Nécessité est mère de l'invention. Sa situation geo-politique difficile amène tout naturellement le Québec à être exceptionnellement créateur sur le plan politique. En cette fin de millénaire où tout bascule, il peut devenir un fascinant laboratoire d'où émergera un nouveau concept politique dont l'application tendra à se généraliser dans ces régions où diverses identités nationales sont enchevêtrées ou appelées à le devenir: Afrique du sud, Yougoslavie, Tchécoslovaquie, Europe en général.

Dans cette optique, la société distincte, loin de constituer une régression par rapport à l'Etat-nation, peut devenir une exceptionnelle percée d'avenir. Le Québec peut être la première société distincte, dans un monde où celles-ci auront davantage d'avenir que bien de traditionnelles nations. La socièté distincte deviendrait l'une des formes politiques avancées de la nation, à l'aube de l'an deux mille.

Dans une perspective historique et mondiale, une querelle entre des Français et des Anglais au nord de l'Amérique renferme des éléments évidents de modernité. Cest au Québec que se vit, dans l'environnement politique le plus évolué, le problème auquel seront invariablement confrontés tous les peuples de la terre: la périlleuse rencontre entre les incontournables enracinements identitaires et l'inévitable intégration politique et économique. Le fiasco yougoslave, de même que l'impuissance de l'Europe à gérer de façon constructive ce conflit, permettent de douter de l'existence ailleurs, d'un modèle applicable aux situations de plus en plus nombreuses où les identités nationales s'interpénètrent. Il n'y a pas vraiment d'exemple étranger dont on puisse s'inspirer.

Pour le meilleur ou pour le pire, le Québec est seul, face à son destin. Mais l'envers de cette angoissante solitude, c'est que les Québécois sont en avance: les premiers, ils pénètrent en territoire nouveau. S'offre à eux une opportunité unique de laisser leur marque, accédant à cet universel auquel ils aspirent. 
En ces domaines, on se réfère toujours à l'exemple européen. Mais, même un projet national aussi éloigné de nous que le projet national indonésien ne pourra éviter certains écueils analogues. En gênéralisant l'usage de la langue malaise, sous le nom de "Bahasa Indonesia", Djakarta entend bien constituer en vraie nation deux cent millions de personnes divisées par les langues, les religions et les coutumes, un ensemble de peuples éparpillés sur dix milles îles. Pour l'instant, si l'on excepte les rebelles de Timor et de la Nouvelle-Guinée occidentale, tout semble fonctionner pour le mieux.

Pourtant, avec l'inéluctable expansion de la démocratie et le développement des médias en langue locale, en particulier de la télévision, nul doute que les paisibles Minangkabaus de Sumatra, les Sassaks de Lombok et les Dayaks de Bornéo approchent de leur moment de cristallisation nationale. Pour empêcher l'explosion tous azimuts, le système politique indonésien sera bien content de disposer d'un concept politique comme celui de société distincte. A moins, bien sûr, que Djakarta ne se cabre. On peut compter sur certains idéologues canadiens pour lui donner là-dessus des leçons de myopie politique.

\section{LE FACTEUR AMÉRICAIN}

Répondant à l'appel de Pierre Elliott Trudeau, certains idéologues canadiens essayèrent de justifier le rejet de l'Accord du lac Meech, cette occasion en or offerte au Canada d'intégrer enfin le phénomène national québécois à un coût minime. Ce n'était pas conforme, dirent-ils, aux principes du fédéralisme. Cela remettait en cause, expliquèrent-ils, rien de moins que les assises de la démocratie libérale.

Sous l'influence américaine, certains penseurs entendent réduire la riche tradition occidentale du libéralisme politique à un système formel et légaliste, dont l'idéalisme n'a d'égale que la stérilité. On doit malheureusement s'attendre à ce que le dogmatisme de ces nouveaux idéologues s'accentue à la suite de l'effondrement du marxisme, le grand concurrent historique du libéralisme politique, version américaine. Dans un bel élan triomphaliste, cette version américaine du libéralisme aura tout naturellement tendance à s'approprier l'ensemble de la tradition libérale occidentale. Elle essaie déjà de réduire cette tradition à la promotion des droits individuels partout dans le monde, se refusant à reconnaitre les incontournables effets politiques des phénomènes nationaux. C'est la recette idéale pour provoquer 
catastrophe sur catastrophe, sous le couvert des grands principes et des bons sentiments.

Dans la version américaine du libéralisme, les faits nationaux deviennent tous plus ou moins analogues à ces phénomènes ethniques qui ont si profondément marqué l'expérience américaine. On considère ces faits nationaux engagés dans un processus de modernisation par rapport à une norme qui s'imposera inéluctablement à l'ensemble de l'univers, une norme évidemment américaine... Et ce, juste au moment où les EtatsUnis sont devenus sous plusieurs plans une nation traditionnelle. On est confronté ici à la plus récente manifestation d'une réalité vieille comme le monde: l'impérialisme. Dans un univers où la domination des valeurs américaines n'a jamais été aussi grande, le danger existe que l'on applique une idéologie libérale sans nuances, vidée de son contenu identitaire, aux différents phénomènes nationaux. Le risque est tout particulièrement grand pour le fait national québécois, assis sur les marches mêmes de l'Empire américain.

Comme l'a bien montré l'échec de l'Accord du lac Meech, comme le démontrait plus récemment la proposition fédérale de l'automne I99I, le combat de la société distincte n'est pas gagné au Canada. L'avenir du concept y dépendra de la façon dont les Québécois seront ou non capables de l'imposer, à la faveur de la crise qui s'annonce. Pourquoi ne serait-ce pas leur tâche historique à eux, la partition qu'ils ont à jouer dans le concert des nations? Surtout qu'il existe des raisons d'être confiant quant à l'issue de l'affaire.

L'une des forces de la société distincte est son caractère modėré, faisant appel au gros bon sens. Il est difficile de taxer de fanatiques ceux qui sont prêts à renoncer au statut de nation pour être reconnus comme membres d'une société distincte. Lors de l'échec de l'Accord du lac Meech, il etait rafraîchissant de lire dans les grands magazines amèricains que le « méchant», pour une fois, c'était davantage le Canada anglais que le Québec. Par ailleurs, sur le libéralisme politique, des penseurs réputés dans le monde anglo-saxon défendent des idées plus nuancées que celles dont nous avons parlé plus haut .

Le concept de société distincte permet le maintien d'une relation privilégiée avec le reste du Canada, en ce qu'il rend plus facile au Québec de reconnaître son côté anglais, son côté canadien. Cela apparaît d'autant plus important qu'à beaucoup d'égards, le Canada anglais reste l'allie privilégié du Québec dans 
le contexte nord-américain, dans la mesure où l'on sera capable de mettre un terme à la relation actuelle basée sur la Conquête. Il y a beaucoup de Canadiens anglais qui se sont courageusement battus pour une vraie reconnaissance de la différence québécoise au sein du Canada, lors du débat sur l'Accord du lac Meech. En essayant de réduire la société distincte québécoise à ses èléments culturels, en tentant de la limiter aux francophones, de la dépolitiser et de la folkloriser au maximum, le système politique canadien ne rend service à personne. Prenons quand même acte du début d'enracinement de la société distincte que manifeste une acceptation même limitee du concept, fruit des efforts du Québec depuis cinq ans. Cela ne saurait manquer d'être utile pour l'avenir.

Cela dit, il reste clair que les Québécois doivent imposer leur société distincte: le reste du pays ne la reconnaîtra jamais vaaiment de lui-même. D'ailleurs, cela s'avérerait une erreur, une dangereuse abdication de pouvoir que de trop miser sur une telle reconnaissance. La société distincte québécoise n'existe pas parce que le reste du Canada la reconnaît. Elle existe, et surtout elle existera, dans la mesure où les Québécois la voudront et l'imposeront.

Fait crucial enfin, le concept de société distincte reste compatible avec l'accession du Québec à la pleine souveraineté sur le plan international. Une société peut devenir à ce point distincte que l'indépendance en vient tout naturellement à s'imposer pour elle. À tout le moins, elle éprouvera vite le besoin de nouer des relations avec ces autres sociétés distinctes qui ne manqueront pas de faire leur apparition à travers le monde. Car, si les Québécois s'avèrent incapables de gagner la bataille de la société distincte, il est probable que d'autres peuples prendront la relève et réussiront là où nous aurons échoué. Chose certaine, ce ne seront pas ces innombrables sociétés-distinctes-bidons que s'acharnent à découvrir ces Canadiens qui veulent à tout prix prolonger la Conquête. Qui sait? Cest peut-être par le moyen de la société distincte que le Québec accédera à l'indépendance. Celle-ci ne nous serait alors rendue qu'après que nous y ayons renoncé.

Institut de recherches politiques du Canada 